\title{
The role of fibrinogen and fibrinogen concentrate in cardiac surgery: an international consensus statement from the Haemostasis and Transfusion Scientific Subcommittee of the European Association of Cardiothoracic Anaesthesiology
}

\author{
G. Erdoes, ${ }^{1}$ A. Koster, ${ }^{2}$ M. I. Meesters, ${ }^{3}$ E. Ortmann, ${ }^{4}$ D. Bolliger, ${ }^{5}$ E. Baryshnikova, ${ }^{6}$ \\ A. Ahmed, ${ }^{7}$ M. D. Lance, ${ }^{8}$ H. B. Ravn, ${ }^{9}$ M. Ranucci, ${ }^{10}$ C. von Heymann ${ }^{11}$ and S. Agarwal ${ }^{12}$ \\ 1 Associate Lecturer, Department of Anaesthesiology and Pain Medicine, Inselspital, Bern University Hospital, University \\ of Bern, Switzerland \\ 2 Associate Lecturer, Institute for Anaesthesiology, Heart and Diabetes Centre NRW, Ruhr-University Bochum, Bad \\ Oeynhausen, Germany \\ 3 Consultant, Department of Anaesthesiology, University Medical Centre Utrecht, The Netherlands \\ 4 Consultant, Department of Anaesthesia, Kerckhoff Heart and Lung Centre, Bad Nauheim, Germany \\ 5 Professor, Department of Anaesthesia, Surgical Intensive Care, Prehospital Emergency Medicine, and Pain Therapy, \\ University Hospital Basel, Switzerland \\ 6 Research Associate, 10 Professor, Department of Cardiovascular Anaesthesia and Intensive Care Unit, IRCCS Policlinico \\ San Donato, Milan, Italy \\ 7 Honorary Associate Professor, Department of Anaesthesia, University Hospitals of Leicester NHS Trust, Leicester, UK \\ 8 Professor, Hamad Medical Corporation, HMC, Anaesthesiology, ICU and Peri-operative Medicine, Doha, Qatar \\ 9 Professor, Department of Cardiothoracic Anaesthesiology, Copenhagen University Hospital, Copenhagen, \\ Denmark \\ 11 Professor, Department of Anaesthesia, Intensive Care Medicine, Emergency Medicine and Pain Therapy, Vivantes \\ Klinikum im Friedrichshain, Berlin, Germany \\ 12 Honorary Senior Lecturer, Department of Anaesthesia, Manchester University Hospitals, Manchester, UK
}

\section{Summary}

To date, data regarding the efficacy and safety of administering fibrinogen concentrate in cardiac surgery are limited. Studies are limited by their low sample size and large heterogeneity with regard to the patient population, by the timing of fibrinogen concentrate administration, and by the definition of transfusion trigger and target levels. Assessment of fibrinogen activity using viscoelastic point-of-care testing shortly before or after weaning from cardiopulmonary bypass in patients and procedures with a high risk of bleeding appears to be a rational strategy. In contrast, the use of Clauss fibrinogen test for determination of plasma fibrinogen level can no longer be recommended without restrictions due to its long turnaround time, high inter-assay variability and interference with high heparin levels and fibrin degradation products. Administration of fibrinogen concentrate for maintaining physiological fibrinogen activity in the case of microvascular post-cardiopulmonary bypass bleeding appears to be indicated. The available evidence does not suggest aiming for supranormal levels, however. Use of cryoprecipitate as an alternative to fibrinogen concentrate might be considered to increase plasma fibrinogen levels. Although conclusive evidence is lacking, fibrinogen concentrate does not seem to increase adverse outcomes (i.e., thromboembolic events). Large prospective multi-centre studies are needed to better define the optimal perioperative monitoring tool, transfusion trigger and target levels for fibrinogen replacement in cardiac surgery.

Re-use of this article is permitted in accordance with the Creative Commons Deed, Attribution 2.5, which does not permit commercial exploitation. 
Correspondence to: G. Erdoes

Email: gabor.erdoes@insel.ch

Accepted: 14 August 2019

Keywords: cardiac surgery; cardiopulmonary bypass; fibrinogen; fibrinogen concentrate

Twitter: @GErdoes

\section{Recommendations}

1 Monitor plasma fibrinogen level in bleeding patients.

2 Consider use of viscoelastic tests - instead of the Clauss assay - for the estimation of fibrinogen's contribution to the clot.

3 Consider a reasonable combination of viscoelastic assays reflecting the intrinsic and extrinsic coagulation pathways as well as fibrinogen function.

4 In patients with continuous, significant, non-surgical and microvascular bleeding who exhibit very low plasma fibrinogen levels $(\leq 4-6 \mathrm{~mm}$ of maximum clot firmness in the fibrin-based thromboelastometry assay, FibTEM-MCF), replacement of fibrinogen is strongly recommended. Under the same circumstances with borderline plasma fibrinogen levels $(6-8 \mathrm{~mm}$ of maximum clot firmness in the fibrin-based thromboelastometry assay), replacement of fibrinogen may be considered.

5 When replacing fibrinogen in patients with diffuse postcardiopulmonary bypass bleeding, maintenance of physiological plasma fibrinogen levels $(>9 \mathrm{~mm}$ of maximum clot firmness in the fibrin-based thromboelastometry assay) should be targeted but very high levels (> $14 \mathrm{~mm}$ of maximum clot firmness in the fibrin-based thromboelastometry assay) should be avoided.

\section{Why was this consensus statement developed?}

Current evidence regarding fibrinogen substitution practice in cardiac surgery is weak. Studies are limited by their small sample size and the large heterogeneity of the patient population, timing of administration and the definition of transfusion trigger and target levels. This consensus statement aims to provide pragmatic guidance for the monitoring, diagnosis and administration of fibrinogen concentrate - based on the available literature and international expert knowledge for patients undergoing cardiac surgery with cardiopulmonary bypass.

\section{How does this consensus statement differ from other available guidelines?}

So far, recommendations on fibrinogen substitution practice in the bleeding patient are available from anaesthesiology societies with thresholds referenced to Clauss assays [1]. However, there is a huge variation with regard to the performance of the Clauss method. Methodological weaknesses of the Clauss assay include an overestimation of plasma fibrinogen level in the presence of artificial colloids and imprecision in the presence of fibrinogen split products [2]. There is convincing evidence that values obtained with the Clauss method during cardiac surgery lack reproducibility among institutions. This limits this method for defining fibrinogen transfusion thresholds and target concentrations. In contrast, viscoelastic tests such as rotational thromboelastometry $\left(\right.$ ROTEM $^{\circledR}$; TEM International $\mathrm{GmbH}$, Munich, Germany) and thromboelastography (TEG ${ }^{\mathrm{TM}}$; Haemonetics Corporation, Braintree, MA, USA) appear to provide a standardised, more reliable and valid laboratory tool for monitoring of the fibrinogen contribution to the clot formation. This consensus statement places particular emphasis on the above points by advising cardiac anaesthetists on the diagnosis of hypofibrinogenemia and practical management of fibrinogen substitution in cardiac surgery patients.

\section{Introduction}

Cardiovascular surgery can lead to coagulopathy and severe bleeding, particularly when cardiopulmonary bypass is involved. Excessive haemorrhage, defined as class 3 or 4 bleeding according to the universal definition of perioperative bleeding [3], occurs in up to $10 \%$ of patients. This serious complication [4] leads to increased blood product requirements and increased medical costs, and affects patient outcome $[5,6]$. Over the past few years, the use of fibrinogen concentrate as a first-line treatment in the bleeding patient has been investigated in a variety of clinical scenarios $[7,8]$. Therapy with fibrinogen concentrate has evolved into a targeted, individualised and algorithmbased replacement strategy in bleeding cardiac surgical 
patients [8]. This application of fibrinogen concentrate significantly differs from its original indication as a fixed dose substitution in patients suffering from congenital a- or dysfibrinogenemia. Therefore, the clinical efficacy and drug safety must be carefully evaluated. This paper aims to provide an expert consensus statement on the current role of fibrinogen and fibrinogen concentrates in cardiac surgery.

\section{Methods}

Eleven cardiac anaesthetists from across Europe and one from the Middle East - all active members of the Haemostasis and Transfusion Scientific Subcommittee of the European Association of Cardiothoracic Anaesthesiology (EACTA) - were invited to participate in this consensus document. A preliminary literature search was performed in the databases PubMed, Cochrane, Science Direct and Google Scholar based on the population, intervention, comparison, outcome and time format [9].

After study selection and quality assessment, the full texts of the retrieved papers were sent to all participants by e-mail. Subsequently, the core topics of this manuscript were determined and survey questions were sent out to the participants focussing on the following domains: (1) monitoring techniques of plasma fibrinogen level during cardiac surgery; (2) timing of plasma fibrinogen level determination in relation to the cardiopulmonary bypass period; (3) trigger and target levels in the treatment of hypofibrinogenemia; (4) institutional practice of fibrinogen administration; (5) preferred usage of concentrated fibrinogen or cryoprecipitate for fibrinogen substitution; (6) practice of overcorrection of plasma fibrinogen levels aiming for high postoperative values; and (7) complications associated with fibrinogen administration. Members of the expert group were asked to express their opinions on each question using the five descriptors 'strongly agree' 'agree', 'neutral', 'disagree' and 'strongly disagree', using a Delphibased method [10]. Consensus was judged to have been achieved if at least two-thirds of the participants were of the same opinion. Items that did not achieve consensus in the first round were either discussed in two additional rounds as a maximum, or rephrased until a consensus could be reached. Chapters were written by members who did not have any conflicts of interest for the specific sub-topic. The entire process lasted eight months.

The literature search identified articles published between September 1993 and October 2018. Overall, 1099 articles were screened. In the first selection, 970 studies were excluded based on title evaluation and lack of relevance to fibrinogen in cardiac surgery. Of the remaining 129 publications, 44 were found to evaluate the clinical effect, monitoring and side-effects of fibrinogen administration in cardiac surgery. After exclusion of duplicates and studies with non-clinical outcomes $(n=17)$, 27 studies (13 randomised controlled trials (RCTs) and 14 non-randomised studies) were found to be relevant. The literature selection process is depicted in detail in Figure 1.

\section{The role of fibrinogen in haemostasis}

Fibrinogen is a hepatically synthesised homodimer glycoprotein consisting of $2 \mathrm{~A} \alpha, 2 \mathrm{~B} \beta$ and $2 \gamma$ polypeptide chains linked by 29 disulphide bridges, with a half-life of 3 5 days. The molecular weight of full-length fibrinogen is $340 \mathrm{kDa}$ and represents $75 \%$ of the plasma fibrinogen [11]. Degraded low molecular weight forms (305 or $270 \mathrm{kDa}$ ) represent approximately $25 \%$ of the circulating fibrinogen and are less effective in promoting the clotting process. Fibrinogen binds the integrin receptor ( $\alpha$ llb $\beta 3$ ) - also called glycoprotein IIb/IIla - on activated platelets, thereby acting as a bridge between thrombocytes in order to facilitate platelet aggregation. Thrombin then cleaves fibrinopeptides from the $A \alpha$ and $B \beta$ chains to form soluble fibrin monomers. Once enzymatically cross-linked under the effect of thrombin-activated factor (F) XIII and due to the binding of a2-antiplasmin, the fibrin clot is relatively resistant to fibrinolysis, ensuring long-term stability and allowing repair of the damaged vessel [12]. The physiological plasma concentration of fibrinogen in non-pregnant patients is 2-4 g. $\mathrm{I}^{-1}$ [13]. This is increased in inflammatory states and during pregnancy and can fall to critically low levels (<1.0 g. $\mathrm{I}^{-1}$ ) during major haemorrhage due to consumption of coagulation factors, dilution and hyperfibrinolysis [14-18].

\section{Fibrinogen-monitoring assays}

Fibrinogen measurement using standard laboratory tests is usually performed in plasma according to the Clauss

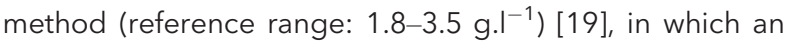
excess of thrombin is added to the diluted sample. The clotting time is measured mechanically or using a photooptical method and compared with a calibration curve. Alternatively an immunological test can be performed to measure the total fibrinogen concentration, rather than the amount of fibrinogen contributing to clotting [20,21].

In a study in five centres in the UK, Mackie et al. assessed the reproducibility of different Clauss assays [22]. Five commercial fibrinogen reference preparations and four commercial reagents for the Clauss assay were used. Additionally, 79 samples taken from patients with different clinical conditions - including disseminated intravascular 


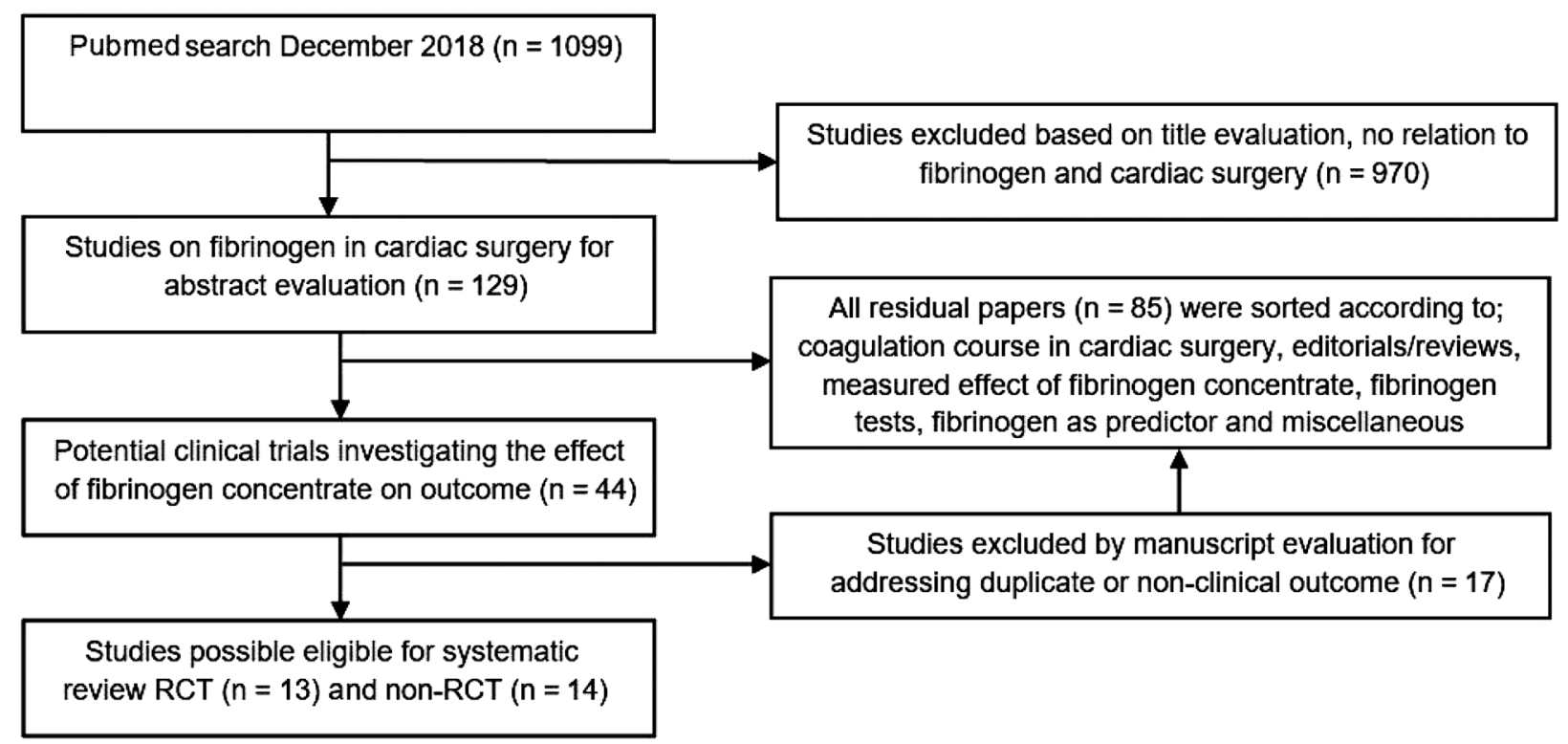

Figure 1 Process used to select literature.

coagulation, severe sepsis, heparin therapy, oral anticoagulation with vitamin $\mathrm{K}$ antagonists and thrombolytic therapy - were evaluated. There was good agreement between centres, analyses and reagents when using the reference preparations, although an increase in scatter was observed with high fibrinogen concentrations. Reproducibility, however, was significantly impaired when analysing samples from patients after thrombolytic therapy or in the presence of disseminated intravascular coagulation.

Point-of-care testing of whole blood with viscoelastic tests can also be used to assess fibrinogen's contribution to a clot. The clot strength is measured with the addition of a potent platelet inhibitor to whole blood, thus blocking the platelet contribution to the clot. In the fibrin-based thromboelastometry assay (FibTEM) for the rotational thromboelastometry device, cytochalasin $D$ is used to inhibit the platelet contribution to the maximum clot firmness (MCF), whereas in the functional fibrinogen assay in thromboelastography, the platelet glycoprotein Ilb/IIla inhibitor abciximab is used to block the platelet effect on the maximum amplitude (MA). For the maximum clot firmness in the fibrin-based thromboelastometry assay, the reference range of 9-25 $\mathrm{mm}$ has been validated in a multicentre trial using samples from healthy volunteers [23], whereas in the functional fibrinogen assay in thromboelastography, the reference range of $11-24 \mathrm{~mm}$ is provided by the manufacturer.

The resulting clot strength is predominantly fibrin based, although it may be partially affected by other conditions, such as deficiencies of factor VIII, factor XIII and von Willebrand factor [24]. Besides a minor effect of the haematocrit on viscoelastic test-derived fibrinogen values [25], residual platelet function may result in 'platelet noise', which overestimates the measured fibrinogen concentration [26]. In a head-to-head comparison of the two methods, fibrin-based thromboelastometry assay and functional fibrinogen assay in thromboelastography, the fibrinogen-based clot amplitudes were found to be higher in the thromboelastography assay [27]. This may be because platelets are less blocked in the functional fibrinogen assay in thromboelastography, leading to more 'platelet noise' and concomitant platelet contribution to a fibrin-based clot formation. The most accurate way of assessing the fibrinogen-based clot appears to be the use of assays with powerful two-way platelet inhibition (e.g. cytochalasin D and glycoprotein Ilb/llla receptor inhibitor) [28]. However, these are not yet universally available.

\section{Cardiac surgery}

Data about the use of standard laboratory tests during cardiopulmonary bypass (hereinafter referred to as 'bypass'), with the aim of predicting fibrinogen levels after weaning from bypass are scarce. A multicentre study in six quality-controlled and specialised laboratories compared the reproducibility and agreement of fibrinogen determination using identical samples and different Clauss methods before surgery, shortly before weaning from bypass and after protamine administration. There was good reproducibility in duplicate measurements, and no 
significant changes in the measured fibrinogen concentration when comparing bypass and postbypass values, suggesting that the results are valid even in the presence of high heparin concentrations. However, there were substantial differences between centres' assessments [20]. The comparability of the various Clauss assays was good when measuring fibrinogen concentration in the range from 4.5 to $12.8 \mathrm{~g} . \mathrm{I}^{-1}$. Additionally, measurements scattered less when centres utilising the same methodology were compared. In particular, the four centres using the photo-optical method demonstrated values ranging from 6.4 to $8.5 \mathrm{~g} . \mathrm{I}^{-1}$, whereas the two centres using mechanical assays obtained fibrinogen levels ranging from 10.4 to $12.8 \mathrm{~g} . \mathrm{I}^{-1}$ [20].

A large retrospective investigation in 1077 cardiac surgery patients demonstrated acceptable correlation $(r=0.78)$ of fibrin-based thromboelastometry assay results with the Clauss assay [29]. A large number of similarly designed studies comparing viscoelastic tests and the standard laboratory tests have been published over recent years [30]. However, due to the lack of reproducibility with different Clauss assays during cardiac surgery with bypass, it remains questionable whether a good correlation of viscoelastic tests with the Clauss assay provides any clinically meaningful information.

\section{Monitoring the effect of transfused fibrinogen concentrate}

In-vitro studies have been performed to assess the effect of fibrinogen concentrate on clot formation and clot strength of the fibrin-based thromboelastometry assay. Blood samples obtained from postoperative cardiac surgery patients were mixed with increasing amounts of fibrinogen concentrate, ranging from 0.5 to $1.5 \mathrm{~g} . \mathrm{I}^{-1}$ [31]. The addition of fibrinogen resulted in a near-linear increase - approximately $6 \mathrm{~mm}$ per $0.5 \mathrm{~g}^{-\mathrm{I}^{-1}}$ fibrinogen - in the maximum clot firmness of the fibrin-based thromboelastometry assay. Comparison of the fibrin-based thromboelastometry assay with different mechanical Clauss assays (mechanical, electromechanical and photo-optical) using samples obtained from 33 patients after bypass, but before fibrinogen concentrate transfusion, showed good correlation ( $r=0.71-0.82)$ [11]. However, similar to results in the aforementioned multicentre study [20], agreement between the different Clauss methods was mostly poor. The correlation between the maximum clot firmness in the fibrin-based thromboelastometry assay and Clauss assays, however, was severely impaired ( $r=0.33$ 0.59) after fibrinogen concentrate transfusion. Additionally, the agreement between the different Clauss assays further decreased. The exact mechanism behind this observation is unclear. High fibrinogen levels appear to affect the precision and reducibility of various Clauss assays [26]. Additionally, endogenous fibrinogen displays a variety of different molecular weights, which have distinct effects on clot formation in the different analysers and reagents used with the Clauss methods [11]. The composition of fibrinogen concentrate might differ from individual endogenous fibrinogen and thereby explain further scattering of test results. Thus the best way of monitoring the efficacy of giving fibrinogen concentrate in cardiac surgery is still not known.

\section{Quality of concentrated fibrinogen}

Fibrinogen can be substituted by transfusion of either cryoprecipitate or fibrinogen concentrate. The relatively low concentration of fibrinogen in fresh frozen plasma (FFP) of 2.5-3.0 g..$^{-1}$ [32] (or even less: 0.9-2.0 $\mathrm{g.l} \mathrm{I}^{-1}$, [33]) after thawing makes fresh frozen plasma unsuitable for fibrinogen replenishment. Cryoprecipitate comprises high molecular weight proteins that are precipitated by thawing leukodepleted fresh frozen plasma from male donors, which is centrifuged and resuspended in 10-20-ml aliquots of plasma [34]. The fibrinogen concentration in cryoprecipitate normally varies between $15 \mathrm{~g}^{-\mathrm{I}^{-1}}$ and

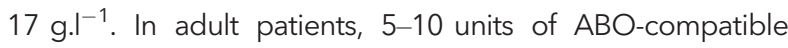
cryoprecipitate are pooled before transfusion. Generally, two units of cryoprecipitate per $10 \mathrm{~kg}$ of body weight raise the plasma fibrinogen concentration by $1 \mathrm{~g} . \mathrm{I}^{-1}$. The risk of viral transmission and adverse immune reactions such as transfusion-related lung injury is very low in modern practice and comparable with fresh frozen plasma [34]

Fibrinogen concentrate is a virus-inactivated derivative of pooled plasma available from different manufacturers and initially produced to correct congenital hypofibrinogenemia, afibrinogenemia or dysfibrinogenemia (Table 1). Reconstitution in sterile water, as manufacturers' guidelines, leads to a concentration of $20 \mathrm{~g}^{-\mathrm{I}^{-1}}$ [34]. Haemocomplettan ${ }^{\circledR}$ (CSL Behring, King of Prussia, PA, USA) and Fibryga ${ }^{\circledR}$ (Octapharma, Lachen, Switzerland) appear to be comparable in terms of pharmacokinetics, safety and clinical efficacy in improving clot firmness in dilutional hypofibrinogenemia [35, 36]. The higher concentration of factor XIII in Fibryga ${ }^{\circledR}(0.2 \mathrm{IU}$ factor XIII per mg fibrinogen) [37] may explain the increased maximum clot firmness observed with thromboelastometry, as demonstrated by in-vitro studies comparing the two products [36]. [Correction added on 5 November 2019, after first online publication: first sentence of this paragraph has been updated.]

When comparing cryoprecipitate with fibrinogen concentrate, it is important to note that fibrinogen is an acute phase protein with significant interindividual 
Table 1 Fibrinogen concentrates on the market, including their indications and availability. Prices may differ considerably according to countries, regions and hospitals and hence costs are only approximate. [Correction added on 5 November 2019, after first online publication: Table 1 has been updated.]

\begin{tabular}{|c|c|c|c|c|}
\hline Trade Name & Manufacturer & Indications & Availability & Costs \\
\hline Haemocomplettan & CSL Behring & $\begin{array}{l}\text { - Treatment and prophylaxis of } \\
\text { acquired and congenital } \\
\text { fibrinogen deficiency } \\
\text { - Acquired hypofibrinogenemia } \\
\text { resulting from severe liver } \\
\text { parenchymal disease and } \\
\text { increased intravascular } \\
\text { consumption }\end{array}$ & $\begin{array}{l}\text { Europe: Austria, Bulgaria, } \\
\text { Czech Republic, } \\
\text { Germany, Hungary, } \\
\text { Portugal, Romania, } \\
\text { Switzerland, Taiwan, } \\
\text { Turkey, The Netherlands } \\
\text { ROW: Argentina, Brazil, } \\
\text { Iran, Israel, Kuwait }\end{array}$ & $\begin{array}{l}469 \cup \operatorname{UD}(1 \mathrm{~g}) \\
896 \operatorname{USD}(2 \mathrm{~g})\end{array}$ \\
\hline Riastap & CSL Behring & $\begin{array}{l}\text { - Treatment of acute bleeding } \\
\text { episodes in patients with } \\
\text { congenital fibrinogen deficiency, } \\
\text { including afibrinogenemia and } \\
\text { hypofibrinogenemia }\end{array}$ & $\begin{array}{l}\text { Europe: Belgium, Cyprus, } \\
\text { Denmark, Finland, } \\
\text { France, Germany, Great } \\
\text { Britain, Greece, Iceland, } \\
\text { Ireland, Italy, } \\
\text { Luxembourg, Malta, } \\
\text { Norway, Poland, Slovak } \\
\text { Republic, Slovenia, Spain, } \\
\text { Sweden } \\
\text { ROW: Australia, Canada, } \\
\text { Mexico, New Zealand, } \\
\text { Puerto Rico, US }\end{array}$ & 486 USD $(1 \mathrm{~g})$ \\
\hline $\begin{array}{l}\text { Fibrinogen } \\
\text { HTBenesis }\end{array}$ & JBPO & $\begin{array}{l}\text { Treatment of bleeding in patients with } \\
\text { congenital fibrinogen deficiency }\end{array}$ & Japan & n.a. \\
\hline $\begin{array}{l}\text { FabuLaishi/ } \\
\text { FibroRAAS }\end{array}$ & Shanghai RAAS & $\begin{array}{l}\text { - Haemorrhage associated with } \\
\text { congenital hypofibrinogenemia, } \\
\text { dysfibrinogenemia or } \\
\text { afibrinogenemia } \\
\text { - Acquired hypofibrinogenemia } \\
\text { due to failure of fibrinogen } \\
\text { synthesis in severe liver disorders } \\
\text { and in increased intravascular } \\
\text { consumption of fibrinogen in DIC } \\
\text { and hyperfibrinolysis }\end{array}$ & China, Japan & n.a. \\
\hline Fibryga & Octapharma & - Congenital fibrinogen deficiency & $\begin{array}{l}\text { Europe: Austria, Belgium, } \\
\text { Czech Republic, } \\
\text { Denmark, Finland, } \\
\text { Germany, Hungary, } \\
\text { Luxembourg, Norway, } \\
\text { Poland, Slovakia, } \\
\text { Slovenia, Sweden, UK } \\
\text { ROW: US, Canada }\end{array}$ & 524 USD (1 g) \\
\hline Clottafact & LFB Biomedicaments & $\begin{array}{l}\text { - Treatment and prophylaxis of } \\
\text { bleeding in patients with } \\
\text { congenital hypo-, dys- or } \\
\text { afibrinogaenemia with bleeding } \\
\text { tendency } \\
\text { - As a complementary therapy for } \\
\text { the treatment of uncontrolled } \\
\text { severe bleeding in acquired } \\
\text { hypofibrinogenemia }\end{array}$ & $\begin{array}{l}\text { Europe: Switzerland, } \\
\text { France } \\
\text { ROW: Algeria, UAE, } \\
\text { Kuwait, Lebanon, } \\
\text { Marocco, Mexico }\end{array}$ & 775 USD (1.5 g) \\
\hline FibCLOT & LFB Biomedicaments & $\begin{array}{l}\text { Treatment and perioperative } \\
\text { prophylaxis of bleeding in patients with } \\
\text { congenital hypo- or afibrinogenaemia } \\
\text { with bleeding tendency }\end{array}$ & $\begin{array}{l}\text { Austria, Belgium, Czech } \\
\text { Republic, Denmark, } \\
\text { Finland, Germany, } \\
\text { Greece, Hungary, Italy, } \\
\text { Luxembourg, Norway, } \\
\text { Slovakia, Spain, Sweden } \\
\text { the Netherlands, UK }\end{array}$ & n.a. \\
\hline
\end{tabular}

ROW, rest of the world; DIC, disseminated intravascular coagulation; US, United States; USD, United States Dollar; n.a., not available. 
variations in plasma concentration. In this respect, a concentrated plasma product may provide a more predictable amount of fibrinogen than cryoprecipitate pooled from 5 to 10 donors. However, cryoprecipitate contains not only concentrated fibrinogen but also von Willebrand factor, factor VIII and factor XIII. A recent systematic review outlined a possible increase in functional improvement of haemostasis with the use of cryoprecipitate [34]. Both clot strength and coagulation activation increased with cryoprecipitate, whereas fibrinogen concentrate only improved clot strength. However, data were limited to one small RCT $(n=63)$ and three observational studies ( $n=218$ ), thus precluding a clear recommendation for one medication over the other.

\section{Treatment of hypofibrinogenemia with fibrinogen concentrates}

Administration of cryoprecipitate or fibrinogen concentrate is recommended as the initial treatment for hypofibrinogenemia in bleeding patients [38]. Several studies have investigated the effect of fibrinogen concentrate on the plasma fibrinogen level (Fig. 2 and Data S1). For every gram of fibrinogen concentrate, there is a $0.2-0.3 \mathrm{~g}^{-1} \mathrm{I}^{-1}$ increase in the Clauss fibrinogen level and a 2-mm increase in the fibrin-based thromboelastometry assay, respectively. The discrepancy in the increase in plasma fibrinogen level between studies may be related to the timing of the fibrinogen measurement; an increase in the time between the administration of fibrinogen concentrate and blood sampling will cause a smaller increase in the fibrinogen level, as fibrinogen is consumed and/or lost due to bleeding. It has also been shown that there are differences between fibrinogen assays [39, 40] and more importantly that the correlation between Clauss fibrinogen and the fibrin-based thromboelastometry assay becomes weaker after administration of the drug [41] or even disappears after fibrinogen concentrate administration $[11,26]$. This may have implications for further fibrinogen supplementation using a fixed threshold [42].

In clinical practice, determination of fibrinogen activity before weaning the patient from bypass may be considered when the risk of bleeding is higher (high-risk patient population or complex cardiac surgery). In cases of persistent microvascular haemorrhage after protamine administration, haemostatic capacity should be assessed using a reasonable combination of viscoelastic assays testing extrinsic and intrinsic activation of the coagulation system, fibrinogen contribution and the effect of residual heparin to cover a large number of common haemostatic disturbances. For rotational thromboelastometry, this could usefully include both the fibrin-based and heparinase assays; when thromboelastography is used, this could include kaolin, kaolin with heparinase, the functional fibrinogen assay and rapid thromboelastography with tissue factor activation.

\section{Drug efficacy}

A systematic review of the efficacy of fibrinogen concentrate in bleeding patients after cardiac surgery reported that
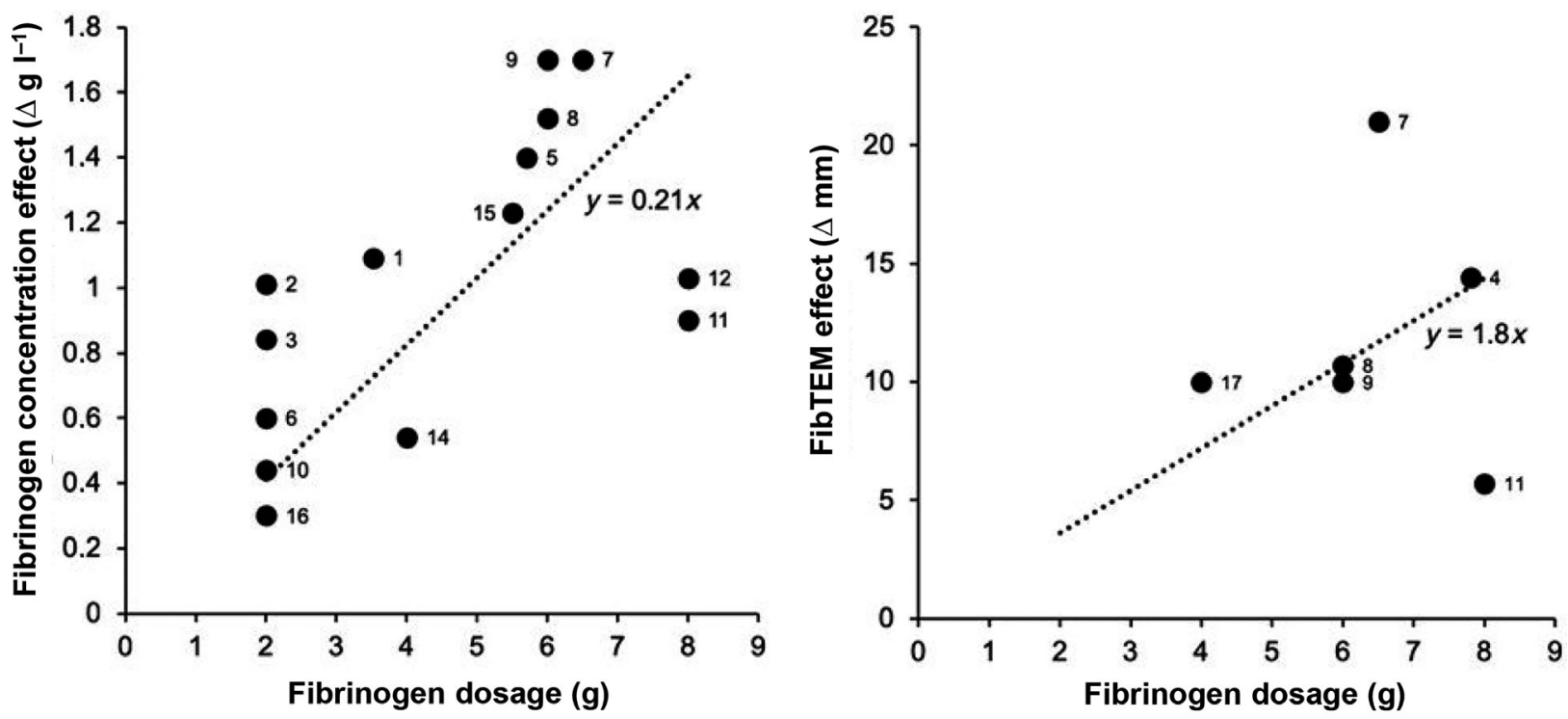

Figure 2 The effect of fibrinogen concentrate on the fibrinogen level in cardiac surgery patients. The left panel shows the effect on Clauss fibrinogen assay, right panel shows the effect on the fibrin-based thromboelastometry assay. The numbers represent the individual studies found in Data S1 (Studies evaluating the effect of fibrinogen concentrate on the plasma fibrinogen level). 
there was no statistically significant effect on mortality, but a significant reduction in blood transfusion requirements [43]. However, the authors also stated that the quality of studies was low, the risk of bias was high and that studies were under-powered to detect mortality. A more recent metaanalysis including eight RCTs in adults undergoing cardiovascular surgery confirmed that the administration of fibrinogen concentrate did not affect all-cause mortality, but confirmed the previously observed reduction in transfusion requirements [8]. However, the eight included trials were small and heterogeneous, with one multicentre [44] and seven single-centre studies including 597 patients, 301 of whom received fibrinogen concentrate. The study protocols varied considerably: in some studies, patients receiving the fibrinogen concentrate were pre-defined before surgery; in other studies, persistent bleeding triggered fibrinogen concentrate therapy. Furthermore, the dose of fibrinogen concentrate was pre-defined (1-2 $\mathrm{g}$ in some studies), whereas other trials prescribed a target level $(22 \mathrm{~mm})$ to be achieved in the fibrin-based thromboelastometry assay, which on average led to transfusion of 4-8 $\mathrm{g}$ of fibrinogen concentrate.

Most RCTs confirm the association of low pre-operative fibrinogen levels with postoperative bleeding [45], although the level of association is weak to moderate and characterised by low positive predictive values [46]. However, the prophylactic correction of pre-operative fibrinogen levels may result in a high rate of inappropriate treatment [46]. In contrast, early postoperative fibrinogen levels are independently associated with postoperative bleeding and the association is stronger than with preoperative fibrinogen levels [43, 46-49].

Studies of the best combination of sensitivity and specificity, in order to define the postoperative fibrinogen level associated with bleeding, established cut-off values ranging from 1.44 to $2.85 \mathrm{~g} . \mathrm{I}^{-1}$ [46-49]. Karkouti et al. found in a predictive model for excessive postbypass bleeding a

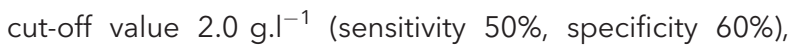
whereas Kindo et al. reported similar data with a cut-off

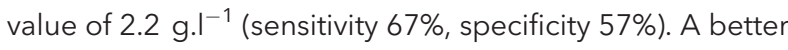
estimation of trigger values for fibrinogen supplementation in case of excessive bleeding is, however, the assessment of a positive predictive value $[50,51]$. Nuttall et al. found that a postoperative fibrinogen level of $1.44 \mathrm{~g} . \mathrm{I}^{-1}$ yielded a good $72 \%$ positive predictive value [47], whereas Ranucci et al. reported a positive predictive value of $50 \%$ for a fibrinogen level of $1.15 \mathrm{~g} . \mathrm{I}^{-1}$ in [48], corresponding to a fibrin-based thromboelastometry assay of 4-6 $\mathrm{mm}$. These values should be considered dynamic in an actively bleeding patient, where fibrinogen levels are declining as fluid is administered and blood loss continues. In the bleeding patient, a Clauss fibrinogen trigger value of $1.5 \mathrm{~g} . \mathrm{I}^{-1}$ is widely accepted (Data S1). In fact, in a non-interventional study of 100 patients undergoing complex cardiac surgery, Bosch et al. found that fibrinogen levels were significantly associated with excessive postoperative blood loss (> 1000 ml.24 $\left.\mathrm{h}^{-1}\right)[52]$. In this study, a median [IQR] Clauss fibrinogen value of $<1.5 \mathrm{~g} . \mathrm{I}^{-1}[1.4-1.6]$ was the strongest predictor ( $p<0.001$ ), compared with 1.9 g. $\mathrm{I}^{-1}[1.8-2.4]$ for excessive blood loss. However, the median fibrin-based thromboelastometry assay value of $4.5 \mathrm{~mm}$ [3-5] also showed a significant association with excessive postoperative blood loss $(p=0.016)$, compared with $9.0 \mathrm{~mm}$ [7.5$12]$. These results suggest that clear trigger values for postbypass fibrinogen substitution are in the range of $\leq 4-6 \mathrm{~mm}$ of maximum clot firmness in the fibrin-based thromboelastometry assay in the diffusely bleeding patient. However, it appears to be 'grey zone' of 6-8 $\mathrm{mm}$ of maximum clot firmness in the fibrin-based thromboelastometry assay which has been suggested by others as a possible trigger for fibrinogen substitution

[29, 53-55].

Target values for plasma fibrinogen should be characterised by a high negative predictive value, as this level by definition is not associated with bleeding or increased blood loss. Ranucci et al. found that a postoperative fibrinogen level of $2.87 \mathrm{~g}^{-1}$ yielded a negative predictive value of $98 \%$. Previous studies mainly aimed for a target fibrin-based thromboelastometry assay of $22 \mathrm{~mm}[48,49]$, which roughly corresponds to a plasma

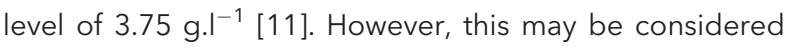
too high, as more recent studies confirmed $14 \mathrm{~mm}$ of maximum clot firmness in the fibrin-based thromboelastometry assay, yielding a $98 \%$ negative predictive value for severe postoperative bleeding [41].

\section{Safety and complications of fibrinogen concentrate administration}

A recent meta-analysis of RCTs assessed the safety of the fibrinogen concentrate in cardiovascular surgery and highlighted the lack of available evidence [8]. The primary outcome of interest was all-cause mortality at study completion. This meta-analysis observed no significant differences in mortality, occurrence of myocardial infarction or stroke between patients receiving fibrinogen or placebo or inactive control (Table 2). However, obvious limitations of included studies (e.g. heterogeneity of safety data) have to be taken into account when interpreting the results. Furthermore, a secondary retrospective subanalysis of a 
Table 2 Fibrinogen administration and safety data of randomised trials [8].

\begin{tabular}{|c|c|c|c|c|c|c|}
\hline \multirow[b]{2}{*}{ Studies } & \multicolumn{2}{|c|}{ Fibrinogen } & \multicolumn{2}{|l|}{ Control $^{a}$} & \multirow[b]{2}{*}{$\mathrm{RR}(95 \% \mathrm{Cl})$} & \multirow[b]{2}{*}{ p } \\
\hline & Patients & Events & Patients & Events & & \\
\hline \multicolumn{7}{|c|}{ Mortality } \\
\hline 5 & 235 & 5 & 12 & 12 & $0.41(0.12-1.38)$ & 0.34 \\
\hline \multicolumn{7}{|c|}{ Myocardial infarction } \\
\hline 4 & 157 & 5 & 160 & 1 & $3.07(0.64-14.78)$ & 1.0 \\
\hline \multicolumn{7}{|l|}{ Stroke } \\
\hline 4 & 225 & 6 & 224 & 7 & $0.82(0.17-4.11)$ & 0.2 \\
\hline
\end{tabular}

$\mathrm{RR}$, relative risk; $\mathrm{Cl}$, confidence interval; values are number or relative risk with confidence interval.

a Only studies with 'placebo' as control.

prospective trial $(n=991)$ analysed the data of 190 patients with fibrinogen concentrate administration (median $2 \mathrm{~g}$ ) and compared them with 190 propensitymatched patients who had not received the fibrinogen concentrate [56]. The primary endpoint was the composite of one-year mortality and major cardiac and thromboembolic events. The secondary endpoint was the composite of death, major cardiac and thromboembolic events within 30 days and all-cause 30-day and 1-year mortality. When groups were compared, it was evident that patients predominantly received the fibrinogen concentrate within the context of major bleeding (30\% vs. $10 \%$ ) and re-operation due to bleeding ( $21 \%$ vs. $7 \%$ ). The unadjusted hazard ratios for primary and secondary adverse outcomes in the fibrinogen concentrate group were between 0.79 (95\% $\mathrm{Cl} 0.39-1.58)$ and $1.46(95 \% \mathrm{Cl} 0.55-3.82)(p=0.678-$ 0.446) compared with matched controls. In the subsequent logistic regression analysis, the use of fibrinogen concentrate was not associated with an increase in mortality or thromboembolic events. In a post-authorisation safety study, Clottafact $^{\circledR}$ (LFB Biomedicaments, France), a human plasma-derived fibrinogen concentrate was evaluated [57]. Amongst 156 total patients, two from the same centre experienced a thromboembolic event (pulmonary embolism/four-site venous thromboembolic episode). Both events were considered to be serious, but a causal relationship with the fibrinogen concentrate could not be established. Rather, the thrombotic events were explained in the context of multiple injuries for one patient and presence of pre-eclampsia for the other. Likewise, a meta-analysis including 14 RCTs $(n=1035)$ in which most patients underwent cardiac surgery could not demonstrate an increase in perioperative thrombotic complications with fibrinogen concentrate [58]. Furthermore, a smaller multicentre retrospective cohort study $(n=1047)$ using propensityscore analyses and multivariate logistic regression analysis investigated the safety of concentrated fibrinogen (fibrinogen/cryoprecipitate) in comparison with placebo (each group $\mathrm{n}=191$ ) and stated that thrombotic events ( 14.1 vs. $14.7 \%, p=0.88$ ) and 30 -day mortality (3.1 vs. $5.2 \%, p=0.30)$ were not different between the groups [59]. It must be borne in mind that all studies have only reported overt clinical thromboembolic events and this does not preclude the presence of silent thrombosis. [Correction added on 5 November 2019, after first online publication: Clottafact ${ }^{\circledR}$ manufacturer details have been updated.]

\section{Concluding recommendations}

In view of the complexity of the haemostatic system, particularly after high-risk cardiac surgery with bypass, it is conceivable that a single pharmacological intervention cannot be exclusively associated with an improvement in clinical outcome due to the heterogeneity of patients, type of surgery, tissue injury, concomitant antiplatelet therapy, etc. Even in an ideal world, large multicentre studies with homogeneous patient populations, reliable laboratory assays, similar peri-operative timing of fibrinogen administration in relation to the bypass period and identical transfusion trigger/target levels would be difficult to establish. Currently, available data are far away from delivering such evidence.

Well-performed, sufficiently powered, prospective multicentre trials addressing the use of fibrinogen concentrate in cardiac surgery are urgently needed. Viewing all available data, it appears that in such studies standardised viscoelastic tests should be used to harmonise fibrinogen monitoring and better define transfusion thresholds. Additionally, such well-designed trials will also help to better evaluate the role of 
viscoelastic tests in the bleeding patient in the special setting of cardiac surgery [60]. Moreover, large sets of safety data are required and should be collected in a standardised manner to improve our experience with the drug/blood product, also in conjunction with the new dosing regimens and high-risk indications during cardiac surgery.

Despite the limitations associated with the available data, we recommend following in fibrinogen monitoring and substitution practice:

1 Current evidence indicates that fibrinogen concentrate may reduce red cell transfusion requirements in cardiac surgery and that restoring plasma physiological fibrinogen levels and activity in a bleeding patient may improve haemostasis. Thus, monitoring plasma fibrinogen level in bleeding patients is a reasonable measure to quickly recognise a fibrinogen-associated haemostasis disorder.

2 We believe that the key to achieving reliable and reproducible data on the effectiveness of fibrinogen replacement therapy is a standardised laboratory environment. The definition of transfusion triggers for fibrinogen supplementation, and evaluation of the effectiveness of the intervention, requires reliable and reproducible assays which provide close agreement in the interpretation of test results. Conditions for monitoring fibrinogen in cardiac surgery, particularly when employing bypass, are unique. The inter-assay variability in the standard laboratory test for fibrinogen, the Clauss assay, is quite large under these conditions, particularly when different detection methods are compared. Additionally, high heparin levels during bypass, fibrin degradation products, polymerisation inhibitors and inhibitors of fibrin formation may affect the reproducibility of test results [61]. After transfusion of the fibrinogen concentrate, reproducibility is limited further. In this regard, whether the Clauss assay can continue to be used as the gold standard for the determination of fibrinogen concentration and for the definition of transfusion triggers in the setting of cardiovascular surgery should be called into question. Additionally, in routine clinical practice, the relatively long turnaround time of the Clauss assay also has to be considered as a substantial limitation in this very dynamic setting. In contrast, the current viscoelastic tests provide standardised reagents and methods for clot detection, as well as computerisation of results, which are then quickly available for the clinician. Although viscoelastic tests are affected by factors other than the fibrinogen plasma concentration, they appear to provide a fair estimation of the fibrinogen activity and contribution to the clot, even during most conditions of cardiac surgery with bypass. Therefore, it appears that the fibrin-based thromboelastometry assay or functional fibrinogen assay in thromboelastography have the potential to be used - instead of the Clauss assay - as the new reference standard in cardiovascular surgery for defining critically low fibrinogen concentrations and for assessing the effectiveness of targeted fibrinogen replacement therapy. However, further studies in the special setting of cardiac surgery should validate 'critical' values of functional fibrinogen assay in thromboelastography corresponding to values of fibrinbased thromboelastometry assay.

3 The fibrin-based thromboelastometry assay or functional fibrinogen assay in thromboelastography are best combined with other viscoelastic assays to cover a range of haemostatic disturbances. For rotational thromboelastometry, this could usefully include both the fibrin-based and heparinase assays; when thromboelastography is used, this could include kaolin, kaolin with heparinase, the functional fibrinogen assay and rapid thromboelastography with tissue factor activation.

4 According to available data, while being aware of the substantial limitations of the quality of these data, a maximum clot firmness in the fibrin-based thromboelastometry assay of $\leq 4-6 \mathrm{~mm}$ should be considered a reasonable trigger in the bleeding patient, whereas a maximum clot firmness in the fibrinbased thromboelastometry assay of 6-8 $\mathrm{mm}$ appears to be a 'grey zone' where considering replacement of fibrinogen may be considered.

5 In post-bypass bleeding, the maintenance of physiological plasma fibrinogen levels (>9 $\mathrm{mm}$ of maximum clot firmness in the fibrin-based thromboelastometry assay) is crucial. The target level is uncertain, but available evidence does not support aiming for supranormal levels (maximum clot firmness in the fibrin-based thromboelastometry assay $>14 \mathrm{~mm}$ ).

\section{Acknowledgements}

EB has received consultancy fees from Werfen. AA has received consultancy fees and research funding from Masimo, Nordic Pharma, CSL Behring and Novo Nordisk. ML received payments for consultancy work and lecture fees related to the topic of this article from 
CSL Behring and TEM International. MR has received consultancy fees and research funding from Roche Diagnostics, CSL Behring, TEM International and Haemonetics. $\mathrm{CvH}$ has received payments for consultancy work and lecture fees related to the topic of this article from CSL Behring and Haemonetics. SA has received research funding and consultancy fees from Haemonetics, Roche, Octapharma and CSL Behring. She is an Editor of Anaesthesia and so the work was sent out for external peer review. The other authors declare that they have no competing interests relevant to this publication.

\section{References}

1. American Society of Anesthesiologists Task Force on Perioperative Blood Transfusion and Adjuvant Therapies. Practice guidelines for peri-operative blood transfusion and adjuvant therapies. Anesthesiology 2006; 105: 198-208.

2. Spahn DR, Bouillon B, Cerny V, et al. The European guideline on management of major bleeding and coagulopathy following trauma: fifth edition. Critical Care 2019; 23: 98.

3. Dyke C, Aronson S, Dietrich W, et al. Universal definition of peri-operative bleeding in adult cardiac surgery. Journal of Thoracic and Cardiovascular Surgery 2014; 147: 1458-63; e1.

4. Ranucci M, Baryshnikova E, Castelvecchio S, Pelissero G; Surgical and Clinical Outcome Research (SCORE) Group. Major bleeding, transfusions, and anemia: the deadly triad of cardiac surgery. Annals of Thoracic Surgery 2013; 96: 478-85

5. Christensen MC, Krapf S, Kempel A, von Heymann C. Costs of excessive postoperative hemorrhage in cardiac surgery. Journal of Thoracic and Cardiovascular Surgery 2009; 138: 687-93.

6. Christensen MC, Dziewior F, Kempel A, von Heymann C. Increased chest tube drainage is independently associated with adverse outcome after cardiac surgery. Journal of Cardiothoracic and Vascular Anesthesia 2012; 26: 46-51.

7. Mengoli C, Franchini M, Marano G, et al. The use of fibrinogen concentrate for the management of trauma-related bleeding: a systematic review and meta-analysis. Blood Transfusion 2017; 15: 318-24.

8. Li JY, Gong J, Zhu F, et al. Fibrinogen concentrate in cardiovascular surgery: a meta-analysis of randomized controlled trials. Anesthesia and Analgesia 2018; 127: 612-21.

9. Sousa-Uva M, Head SJ, Thielmann M, et al. Methodology manual for European Association for Cardio-Thoracic Surgery (EACTS) clinical guidelines. European Journal of CardioThoracic Surgery 2015; 48: 809-16.

10. McMillan SS, King M, Tully MP. How to use the nominal group and Delphi techniques. International Journal of Clinical Pharmacy 2016; 38: 655-62.

11. Solomon C, Cadamuro J, Ziegler B, et al. A comparison of fibrinogen measurement methods with fibrin clot elasticity assessed by thromboelastometry, before and after administration of fibrinogen concentrate in cardiac surgery patients. Transfusion 2011; 51: 1695-706.

12. Lisman T, de Moerloose P. The role of fibrinogen and factor XIII in hemostasis, and the identification and treatment of associated disorders. Seminars in Thrombosis and Hemostasis 2016; 42: 331-2.

13. Kattula $\mathrm{S}$, Byrnes JR, Wolberg AS. Fibrinogen and fibrin in hemostasis and thrombosis. Arteriosclerosis, Thrombosis, and Vascular Biology 2017; 37: e13-21.
14. Levy JH, Welsby I, Goodnough LT. Fibrinogen as a therapeutic target for bleeding: a review of critical levels and replacement therapy. Transfusion 2014; 54: 1389-405.

15. Maung AA, Kaplan LJ. Role of fibrinogen in massive injury. Minerva Anestesiologica 2014; 80: 89-95.

16. Hiippala ST, Myllylä GJ, Vahtera EM. Hemostatic factors and replacement of major blood loss with plasma-poor red cell concentrates. Anesthesia and Analgesia 1995; 81:360-5.

17. Murray DJ, Pennell BJ, Weinstein SL, Olson JD. Packed red cells in acute blood loss: dilutional coagulopathy as a cause of surgical bleeding. Anesthesia and Analgesia 1995; 80: 336-42.

18. Floccard B, Rugeri L, Faure A, et al. Early coagulopathy in trauma patients: an on-scene and hospital admission study. Injury 2012; 43: 26-32.

19. Tarallo P, Henny J, Gueguen R, Siest G. Reference limits of plasma fibrinogen. European Journal of Clinical Chemistry and Clinical Biochemistry 1992; 30: 745-51.

20. Solomon C, Baryshnikova E, Tripodi A, et al. Fibrinogen measurement in cardiac surgery with cardiopulmonary bypass: analysis of repeatability and agreement of Clauss method within and between six different laboratories. Thrombosis and Haemostasis 2014; 112: 109-17.

21. Mackie IJ, Kitchen S, Machin SJ, Lowe GD; Haemostasis and Thrombosis Task Force of the British Committee for Standards in Haematology. Guidelines on fibrinogen assays. British Journal of Haematology 2003; 121: 396-404.

22. Mackie J, Lawrie AS, Kitchen S, et al. A performance evaluation of commercial fibrinogen reference preparations and assays for Clauss and PT-derived fibrinogen. Thrombosis and Haemostasis 2002; 87: 997-1005.

23. Lang $T$, Bauters $A, B$ raun $S L$, et al. Multi-centre investigation on reference ranges for ROTEM thromboelastometry. Blood Coagulation and Fibrinolysis 2005; 16: 301-10.

24. Schramko AA, Kuitunen AH, Suojaranta-Ylinen RT, Niemi TT. Role of fibrinogen-, factor VIII- and XIII-mediated clot propagation in gelatin haemodilution. Acta Anaesthesiologica Scandinavica 2009; 53: 731-5.

25. Solomon C, Rahe-Meyer N, Schöchl H, Ranucci M, Görlinger K. Effect of haematocrit on fibrin-based clot firmness in the FIBTEM test. Blood Transfusion 2013; 11:412-8.

26. Ogawa S, Szlam F, Bolliger D, Nishimura T, Chen EP, Tanaka KA. The impact of hematocrit on fibrin clot formation assessed by rotational thromboelastometry. Anesthesia and Analgesia 2012; 115: 16-21.

27. Prüller $F$, Münch $A$, Preininger $A$, et al. Comparison of functional fibrinogen (FF/CFF) and FIBTEM in surgical patients - a retrospective study. Clinical Chemistry and Laboratory Medicine 2016; 54: 453-8.

28. Bolliger D, Seeberger MD, Tanaka KA. Principles and practice of thromboelastography in clinical coagulation management and transfusion practice. Transfusion Medicine Reviews 2012; 26: 1-13.

29. Mace H, Lightfoot N, McCluskey $S$, et al. Validity of thromboelastometry for rapid assessment of fibrinogen levels in heparinized samples during cardiac surgery: a retrospective, single-center, observational study. Journal of Cardiothoracic and Vascular Anesthesia 2016; 30: 90-5.

30. Peng HT, Nascimento B, Beckett A. Thromboelastography and thromboelastometry in assessment of fibrinogen deficiency and prediction for transfusion requirement: a descriptive review. BioMed Research International 2018; 2018: 7020539.

31. Shams Hakimi C, Fagerberg Blixter I, Hansson EC, Hesse C, Wallén $H$, Jeppsson A. Effects of fibrinogen and platelet supplementation on clot formation and platelet aggregation in blood samples from cardiac surgery patients. Thrombosis Research 2014; 134: 895-900. 
32. von Heymann C, Keller MK, Spies C, et al. Activity of clotting factors in fresh-frozen plasma during storage at 4 degrees $C$ over 6 days. Transfusion 2009; 49: 913-20.

33. Theusinger OM, Baulig W, Seifert B, Emmert MY, Spahn DR, Asmis LM. Relative concentrations of haemostatic factors and cytokines in solvent/detergent-treated and fresh-frozen plasma. British Journal of Anaesthesia 2011; 106: 505-11.

34. Jensen NH, Stensballe J, Afshari A. Comparing efficacy and safety of fibrinogen concentrate to cryoprecipitate in bleeding patients: a systematic review. Acta Anaesthesiologica Scandinavica 2016; 60: 1033-42.

35. Ross C, Rangarajan S, Karimi M, et al. Pharmacokinetics, clot strength and safety of a new fibrinogen concentrate: randomized comparison with active control in congenital fibrinogen deficiency. Journal of Thrombosis and Haemostasis 2018; 16: 253-61.

36. Haas T, Cushing MM, Asmis LM. Comparison of the efficacy of two human fibrinogen concentrates to treat dilutional coagulopathy in vitro. Scandinavian Journal of Clinical and Laboratory Investigation 2018; 78: 230-5.

37. Schulz PM, Gehringer W, Nöhring S, et al. Biochemical characterization, stability, and pathogen safety of a new fibrinogen concentrate $\left(\right.$ fibryg $\left.{ }^{\circledR}\right)$. Biologicals 2018; 52: 72-7.

38. Task Force on Patient Blood Management for Adult Cardiac Surgery of the European Association for Cardio-Thoracic Surgery (EACTS) and the European Association of Cardiothoracic Anaesthesiology EACTA, Boer C, Meesters MI, et al. 2017 EACTS/EACTA Guidelines on patient blood management for adult cardiac surgery. Journal of Cardiothoracic and Vascular Anesthesia 2018; 32: 88-120.

39. Solomon C, Asmis LM, Spahn DR. Is viscoelastic coagulation monitoring with ROTEM or TEG validated. Scandinavian Journal of Clinical and Laboratory Investigation 2016; 76: 503-7.

40. Fenger-Eriksen C, Moore GW, Rangarajan S, Ingerslev J, Sørensen B. Fibrinogen estimates are influenced by methods of measurement and hemodilution with colloid plasma expanders. Transfusion 2010; 50: 2571-6.

41. Ranucci M, Baryshnikova E. Fibrinogen supplementation after cardiac surgery: insights from the Zero-Plasma trial (ZEPLAST), British Journal of Anaesthesia 2016; 116: 618-23.

42. Weber CF, Görlinger K, Meininger D, et al. Point-of-care testing: a prospective, randomized clinical trial of efficacy in coagulopathic cardiac surgery patients. Anesthesiology 2012; 117: $531-47$.

43. Wikkelsø A, Lunde J, Johansen $M$, et al. Fibrinogen concentrate in bleeding patients. Cochrane Database of Systematic Reviews 2013; CD008864.

44. Rahe-Meyer N, Levy JH, Mazer CD, et al. Randomized evaluation of fibrinogen vs placebo in complex cardiovascular surgery (REPLACE): a double-blind phase III study of haemostatic therapy. British Journal of Anaesthesia 2016; 117: 41-51.

45. Gielen C, Dekkers O, Stijnen T, et al. The effects of pre- and postoperative fibrinogen levels on blood loss after cardiac surgery: a systematic review and meta-analysis. Interactive Cardiovascular and Thoracic Surgery 2014; 18: 292-8.

46. Ranucci M, Jeppsson A, Baryshnikova E. Pre-operative fibrinogen supplementation in cardiac surgery patients: an evaluation of different trigger values. Acta Anaesthesiologica Scandinavica 2015; 59: 427-33.

47. Nuttall GA, Oliver WC, Ereth MH, Santrach PJ. Coagulation tests predict bleeding after cardiopulmonary bypass. Journal of Cardiothoracic and Vascular Anesthesia 1997; 11: 815-23.

48. Ranucci M, Pistuddi V, Baryshnikova E, Colella D, Bianchi P. Fibrinogen levels after cardiac surgical procedures: association with postoperative bleeding, trigger values, and target values. Annals of Thoracic Surgery 2016; 102: 78-85.

49. Rahe-Meyer N, Pichlmaier M, Haverich A, et al. Bleeding management with fibrinogen concentrate targeting a high- normal plasma fibrinogen level: a pilot study. British Journal of Anaesthesia 2009; 102: 785-92.

50. Karkouti K, Callum J, Crowther MA, et al. The relationship between fibrinogen levels after cardiopulmonary bypass and large volume red cell transfusion in cardiac surgery: an observational study. Anesthesia and Analgesia 2013; 117: 14-22.

51. Kindo M, Hoang Minh T, Gerelli S, et al. Plasma fibrinogen level on admission to the intensive care unit is a powerful predictor of postoperative bleeding after cardiac surgery with cardiopulmonary bypass. Thrombosis Research 2014; 134: 360-8.

52. Bosch YPJ, Weerwind PW, Ten Cate $\mathrm{H}$, et al. The association between rotational thromboelastometry parameters and blood loss after cardiac surgery with cardiopulmonary bypass. Cardiovascular and Thoracic Open 2015; 1-8. https://doi.org/ 10.1177/2055552015599644.

53. Karkouti K, McCluskey SA, Callum J, et al. Evaluation of a novel transfusion algorithm employing point-of-care coagulation assays in cardiac surgery: a retrospective cohort study with interrupted time-series analysis. Anesthesiology 2015; 122: 560-70.

54. Kuiper GJAJM, van Egmond LT, Henskens YMC, et al. Shifts of transfusion demand in cardiac surgery after implementation of rotational thromboelastometry-guided transfusion protocols: analysis of the HEROES-CS (HEmostasis Registry of patiEntS in Cardiac Surgery) observational, prospective open cohort database. Journal of Cardiothoracic and Vascular Anesthesia 2019; 33: 307-17.

55. Lehmann F, Rau J, Malcom B, et al. Why does a point of care guided transfusion algorithm not improve blood loss and transfusion practice in patients undergoing high risk cardiac surgery? A prospective randomized controlled pilot study. BMC Anesthesiology 2019; 19: 24.

56. Fassl J, Lurati Buse G, Filipovic $M$, et al. Peri-operative administration of fibrinogen does not increase adverse cardiac and thromboembolic events after cardiac surgery. British Journal of Anaesthesia 2015; 114: 225-34.

57. Négrier C, Ducloy-Bouthors AS, Piriou V, et al. Postauthorization safety study of Clottafact ${ }^{\circledR}$, a triply secured fibrinogen concentrate in acquired fibrinogen deficiency: a prospective observational study. Vox Sanguinis 2018; 113: 120-7.

58. Fominskiy E, Nepomniashchikh VA, Lomivorotov WV, et al. Efficacy and safety of fibrinogen concentrate in surgical patients: a meta-analysis of randomized controlled trials. Journal of Cardiothoracic and Vascular Anesthesia 2016; 30: 1196-204.

59. Maeda T, Miyata S, Usui A, et al. Safety of fibrinogen concentrate and cryoprecipitate in cardiovascular surgery: multicenter database study. Journal of Cardiothoracic and Vascular Anesthesia 2019; 33: 321-7.

60. Wikkelsø A, Wetterslev J, Møller AM, Afshari A. Thromboelastography (TEG) or rotational thromboelastometry (ROTEM) to monitor haemostatic treatment in bleeding patients: a systematic review with meta-analysis and trial sequential analysis. Anaesthesia 2017; 72: 519-31.

61. Koh SC, Chew CY, Viegas OA, Choo M, Ratnam SS. Influence of circulating D-dimer levels on assays of fibrinogen. Annals of the Academy of Medicine, Singapore 1994; 23: 856-60.

\section{Supporting Information}

Additional supporting information may be found online in the Supporting Information section at the end of the article.

Data S1. Studies evaluating the effect of fibrinogen concentrate on the plasma fibrinogen level (part 1) and recommended level of fibrinogen for substitution in different European and US guidelines (part 2). 